\title{
Explorations and Practices on Course Teaching of Computer Graphics Based on Virtual Reality
}

\author{
Zhi Zhao ${ }^{1, a}$, Hui-jie $Q u^{1, b}$ and Jin-de Huang ${ }^{1, c, *}$ \\ ${ }^{1}$ School of Mathematics and Information Science, Guangxi College of Education, Nanning 530023, \\ China \\ aZZz191126.com, bqq_r252@126.com, ch_jinde@tom.com \\ ${ }^{*}$ Corresponding author: Jin-de Huang
}

Keywords: Computer graphics, Algorithm, Virtual experiment, Practices.

\begin{abstract}
Computer graphics, as one of important courses related computer and related disciplines, attaches equal importance to both theories and practices. It is a highly practical preparatory course for digital image pattern recognition and 3D animation programming. In light of problems encountered by students in learning complicated algorithms of computer graphics, this study proposes integrating virtual experiments into teaching, in order that abstract objects and operations in algorithms can be visualized and algorithms can become easier to understand for the final purpose of improving teaching. The results suggest that significant improvements have been observed in Class 16A compared with Class 15A. The average scores have increased to 55.62 points in Class 16A, where the maximum and minimum scores have rose by 11 and 1 respectively, while an $8.1 \%$ increase has been detected in the pass rate. According to related experimental data, classroom teaching has achieved relatively ideal outcomes.
\end{abstract}

\section{Intorduction}

Computer Graphics is an elementary course about computer sciences and application with critical theoretical and practical significance. Its theories and application technologies have been widely used in different fields, including agriculture, teaching, scientific research and military affairs. Meanwhile, its theories and applications are developing. This course is playing more and more important roles in sciences, technologies and human practices. Nevertheless, two major problems are common in teaching this course:

On one hand, Computer Graphics, attaching equal importance to both theories and experiments, covers much content, whereas insufficient credit hours are arranged to teaching of this course.

The classroom teaching of this course, consists of algorithms and experiments about graphics, is performed by approximately 68 credit hours in total. When theoretical and experimental teaching tasks are fulfilled within limited credit hours, classroom teaching may merely focus on elaborating basic concepts, algorithms and thoughts of graphics. Instead, nothing about the backgrounds under which algorithms are generated, mathematical ideas or models of the algorithms, connections among similar algorithms and extensions of teaching may be covered in the classroom teaching. Furthermore, following differences exist in learning between Computer Graphics and other computer courses. Computer Graphics is a discipline that pays equal attention to theories and practices. In learning this course, students do not only have to grasp theoretical thoughts and methods of graphic algorithms, but also have to experimentally validate strengths and weaknesses of these algorithms by writing program codes. The graphic algorithms are often associated with related mathematical thoughts and models that they touch upon much more mathematical knowledge than all other disciplines that students have learnt before, so lots of students feel hard to learn this course.

On the other hand, this course is taught in a boring way that it is impossible to give full play to students' learning autonomy. As a result, the effects of classroom teaching are not quite ideal. At present, Computer Graphics is taught in monotonously traditional ways not in line with requests of teaching objects in many universities, where the course is simply taught from "concepts to experiments". This is associated with so much content and taught within limited credit hours that a 
majority of teachers have no choice but to teach the course fast in a generalized way. However, some students are not adaptable to too fast and generalized classroom teaching. As a consequence, heavy burden and poor efficiency become bottlenecks of the classroom teaching, as a consequence of which the effects of the classroom teaching are extremely worrying.

According to modern pedagogy, learning qualities refer to stable psychological traits of students reflected from their learning behaviors, particularly their learning motivations, attitudes towards learning, learning strategies, wills and characters indicated from learning. Apart from their significance for acquiring knowledge and skills, learning qualities also imply individuals' comprehensive qualities, personalities and tastes. One objective of modern teaching is just to improve students' comprehensive qualities, personalities and tastes. Through this study, efforts are made to develop interactive learning resources for Computer Graphics with virtual reality technologies, and create a classroom teaching model for enabling students to autonomously learn, make explorations, acquire knowledge and play their roles a subjects, so that they may become skilled at obtaining information from smooth channels, develop stronger abilities to search/track information, strengthen their capabilities of absorbing knowledge and get motivated to learn Computer Graphics with great enthusiasm, for the final purpose of bringing their roles as subjects into full play and completely improving their competence for autonomous learning. This study is of critical realistic significance for deepening teaching reform, thoroughly promoting quality-based education, fostering students' statistical thoughts and developing their comprehensive practical skills.

\section{Research Objects and Methods}

In this paper, adult undergraduates who were admitted by the School of Mathematics and Information Science of our university in 2015 were chosen as research objects and examined by new teaching methods to observe the teaching effects.

This study was mainly performed by literature review and experiments on classroom teaching.

With their rapid development over the past years, virtual reality technologies have been extensively utilized in multiple fields. From the perspective of visual effects, 3D scenes are more dynamic and imaginative than 2D planes. As they are developing, virtual reality technologies will be inevitably incorporated into modern education, thus making it possible to apply these technologies in education to improve teaching. Teaching theories and practices based on these technologies will become a new approach to teaching reforms.

Hence, existing teaching model of Computer Graphics must be transformed. To this end, virtual reality technologies just make up for deficiencies of conventional teaching methods, on the grounds that they simulate results of all experimental processes, so that students may promptly understand origins, causes and results of graphic algorithms. In this way, they will not only acquire a better understanding of mathematical models and thoughts related to graphic algorithms, but also can take advantage of virtual reality technologies to simulate experimental environment. At last, breakthroughs may be made in traditional models and methods with advanced network technologies. On one hand, virtual reality technologies further diversify experimental processes, results and details for teaching of Computer Graphics, thereby contributing to students' better understanding of graphic algorithms; on the other hand, they make a practical environment available for students' simulation while realizing highly orderly and massive sharing of teaching related information beyond tempo-spatial limitations of conventional teaching in different parts, so as to ultimately increase teaching efficiency of Computer Graphics through human-computer interactions and people's communications, thus attaining the final goal of improving teaching.

\section{Experimental Processes and Analysis of Classroom Teaching}

As described below, 3D graphs are converted into teaching content of computer graphics, which is taught in combination with virtual reality. The graphic algorithms used for converting 3D graphs are 
presented by virtual methods, and operations of graphic algorithms that are invisible in computers are visualized, so that teachers don't purely describe algorithms of computer graphics in words.

Transformation by rotation around $\mathrm{X}$-axis is one of typical geometric transformation of 3D graphs and its key program codes are shown as follows:

(1) Program codes of 4-order matrix multiplication are as follows:

void CTestView::Calculate(double P0[][4],double T[][4])

\{

double Ptemp[8][4];

KeepOriginalMatrix(P3D,Ptemp);

for(int $\mathrm{i}=0 ; \mathrm{i}<8 ; \mathrm{i}++)$

for(int $\mathrm{j}=0 ; j<4 ; j++)$

Ptemp[i][3]*T[3][j];

$\mathrm{P} 3 \mathrm{D}[\mathrm{i}][\mathrm{j}]=$ Ptemp[i][0]*T[0][j]+Ptemp[i][1]*T[1][j]+Ptemp[i][2]*T[2][j]+

\}

(2) For rotation around $X$-axis, the program codes are as follows:

void CTestView::Txrotate(double thta)

//matrix functions of transformation by rotation around $\mathrm{X}$-axis

\{

ClearMatrix(TXR);

RedrawWindow();

TXR $[0][0]=1$;

TXR[1][1] $=\cos ($ thta*PI/180);

TXR[1][2]=sin(thta*PI/180);

TXR[2][1]=-sin(thta*PI/180);

TXR[2][2] $=\cos ($ thta*PI/180);

TZR[3][3]=1;

Calculate(P3D,TXR);

AfxGetMainWnd()->SetWindowText("geometric transformation of 3D graphs:

transformation by rotation around X-axis");

DrawCube();

\}

(3) Algorithms are realized according to several steps, of which the flowchart is shown as follows:

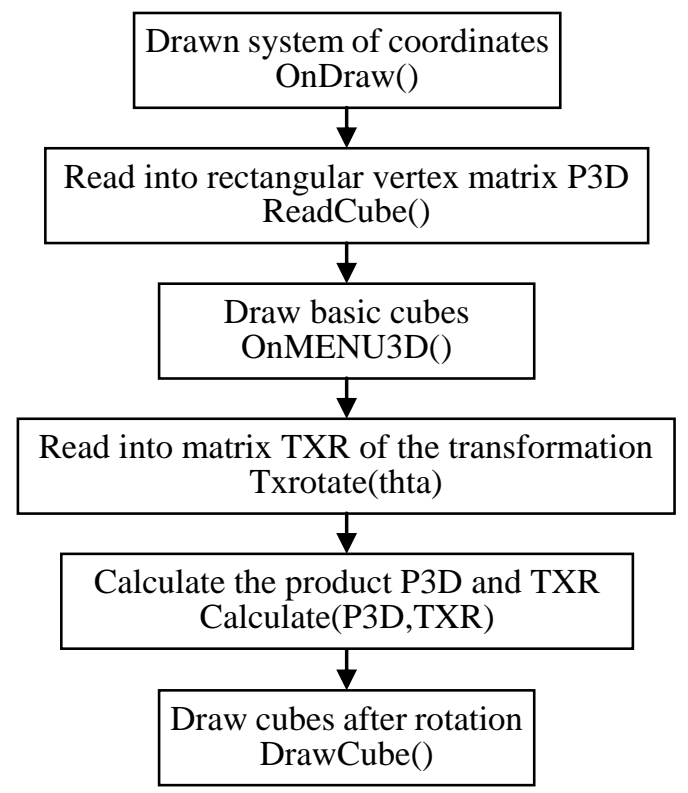

Fig 1. Flowchart on the Algorithm of the Transformation by Rotation around X-axis Fig 1 each step functional specification is as follows. 
Step1: Draw up the coordinate system by the program function OnDraw();

Step2: Read the cube vertices matrix P3D by function ReadCube();

Step3: Draw up the cube basic graph by function OnMENU3D();

Step4: Calculate the product of matrix P3D and TXR by function Calculate(P3D,TXR);

Step5: Draw the cube after rotation by function DrawCube().

(4) Data about Results in Final Term Exams and Comparisons

The final term examination results of classes 15A and 16A from the School of Mathematics and Information Science in computer graphics are described as follows. Of both classes, Class 15A has been taught by traditional teaching methods, while the other class has been taught by novel teaching methods.

Table 1. Final Term Examination Results of Class 16A from the School of Mathematics and Information Science in Computer Graphics

\begin{tabular}{|c|c|c|}
\hline Student ID & Name & Final Term Exam \\
\hline 160222A01001 & Zheng Fuxian & 93 \\
\hline 160222A01002 & Lian Zuyun & 81 \\
\hline $160222 \mathrm{~A} 01003$ & Fang Yuwei & 72 \\
\hline $160222 \mathrm{~A} 01004$ & Zhu Chunyi & 22 \\
\hline $160222 \mathrm{~A} 01005$ & Zhu Anning & 84 \\
\hline $160222 \mathrm{~A} 01006$ & Zheng Chuqian & 74 \\
\hline $160222 \mathrm{~A} 01007$ & Yu Deyue & 67 \\
\hline $160222 \mathrm{~A} 01012$ & Jiao Xingxing & 91 \\
\hline $160222 \mathrm{~A} 01 \mathrm{x} 01$ & He Long & 64 \\
\hline 160222A01x02 & Zhang Xuemin & 32 \\
\hline $160222 \mathrm{~A} 01 \mathrm{x} 03$ & Liang Zhenhua & 52 \\
\hline 160222A01x04 & Ling Jianhui & 76 \\
\hline 160222A01x07 & Huang Zhongman & 96 \\
\hline 160222A01x08 & Yang Hui & 65 \\
\hline 160222A01x09 & Luo Chungui & 76 \\
\hline
\end{tabular}

Table 2. Final Term Examination Results of Class 15A from the School of Mathematics and Information Science in Computer Graphics

\begin{tabular}{|l|l|c|}
\hline Student ID & \multicolumn{1}{|c|}{ Name } & Final Term Exam \\
\hline $150222 \mathrm{~A} 01001$ & Wu Qizhao & 69 \\
\hline $150222 \mathrm{~A} 01002$ & Tan Fangzeng & 60 \\
\hline $150222 \mathrm{~A} 01003$ & Huang Cuiping & 69 \\
\hline $150222 \mathrm{~A} 01004$ & Zhou Zhiting & 65 \\
\hline $150222 \mathrm{~A} 01005$ & Huang Zhenqin & 74 \\
\hline $150222 \mathrm{~A} 01007$ & Feng Lifeng & 55 \\
\hline $150222 \mathrm{~A} 01008$ & Huang Hao & 79 \\
\hline $150222 \mathrm{~A} 01009$ & Fu Yiyao & 54 \\
\hline $150222 \mathrm{~A} 01010$ & Li Binqun & 86 \\
\hline $150222 \mathrm{~A} 01 x 01$ & He Shenping & 73 \\
\hline $150222 \mathrm{~A} 01 \mathrm{x} 02$ & Wei Yubin & 49 \\
\hline $150222 \mathrm{~A} 01 x 03$ & Liang Junchang & 66 \\
\hline $150222 \mathrm{~A} 01 x 04$ & Li Xiaoming & 65 \\
\hline $150222 \mathrm{~A} 01 x 05$ & Li Zhijian & \\
\hline
\end{tabular}


Table 3. Comparative Analysis of Exam Results between Classes 15A and 16A

\begin{tabular}{|c|c|c|c|c|}
\hline & Average Scores & Maximum Scores & Minimum Scores & Pass Rate \\
\hline Class 15A & 67.78 & 85 & 49 & $78.57 \%$ \\
\hline Class 16A & 73.4 & 96 & 50 & $86.67 \%$ \\
\hline
\end{tabular}

The scores are arranged in order from the higher to the lower in Tables 1 and 2, based on which the scores are compared in Fig 2 as follows.

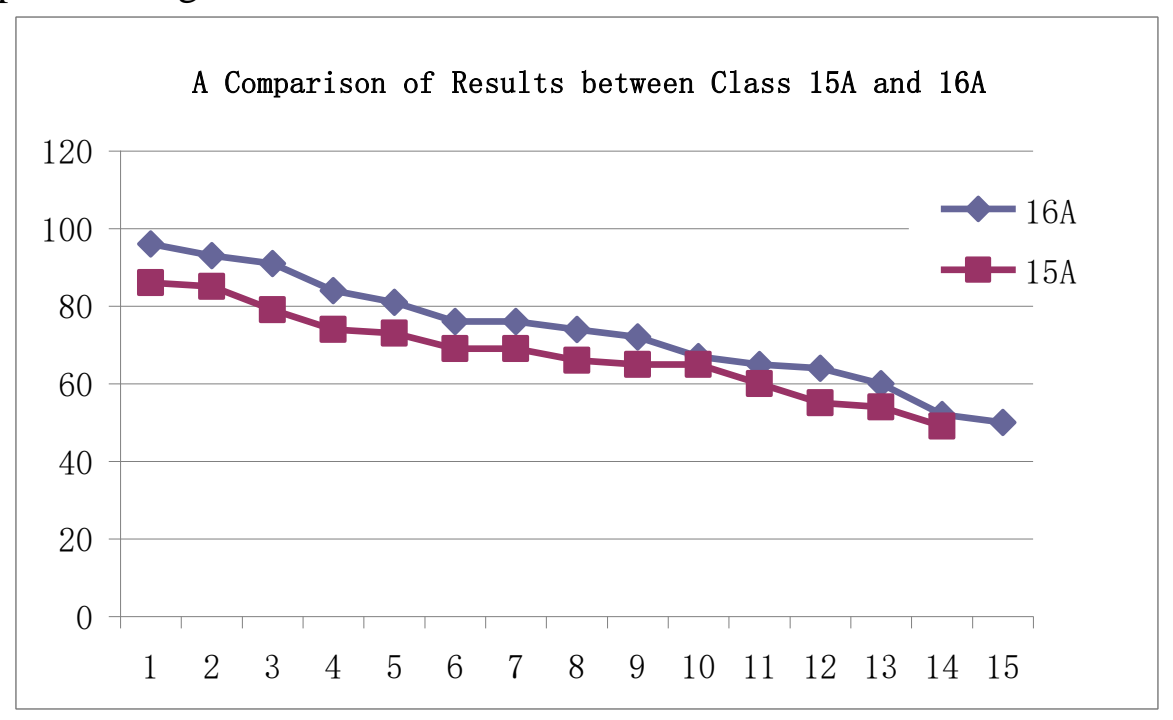

Fig 2. A Comparison of Results between Class 15A and 16A

Tables 1 and 2 show original data about scores.

From Table 3, it may be observed that:

The average scores, maximum scores, minimum scores and pass rate of Class $15 \mathrm{~A}$ in graphics are $67.78,85,49$ and $78.57 \%$ respectively, but $73.4,96,50$ and $86.67 \%$ respectively in Class $16 \mathrm{~A}$. By comparing results of both classes, it is clear that Class 16A outperforms Class 15A in all four parts.

As suggested in Fig 2 (a comparison of results between Class 15A and 16A), the results of both classes are compared after they are arranged in a declining order. Compared with Class 15A, Class $16 \mathrm{~A}$ has made more evident progress in their results.

\section{Conclusions}

In this paper, virtual reality technologies are integrated into the classroom teaching of Computer Graphics as an aided teaching tool, and final exam results of Class 16A are compared with those of Class $15 \mathrm{~A}$, where graphics is taught by conventional teaching methods. The results suggest that Class 16A makes more significant improvements than Class 15A, which thus verifies that the new teaching method proposed in this paper is effective. In Class 16A, the average scores, maximum scores, minimum scores and pass rate have increased to $55.62 \%$, by 11,1 and $8.1 \%$ respectively. Above all, it is one of effective methods for improving students' learning outcomes by incorporating virtual reality technologies into classroom teaching of Computer Graphics. 


\section{Acknowledgement}

This research was financially supported by the Education Department of Guangxi Province (NO.2015JGZ172), and scientific research projects of Guangxi College of Education in 2015 (NO.YB2014587, NO.A2015002), and 2015 Special Program on Hybrid Teaching Reform for Education and Teaching Reform of Guangxi College of Education (NO.XJJG15A03), and 2016 Program about Teaching Teams with "Key Competencies" in Guangxi College of Education, Topic: Research on Integrating Cultivation of Key Competencies into Teaching.

\section{References}

[1] Fu Yuchen, Zhou Dongru. Principles, Methods and Applications of Computer Graphics, Huazhong University of Science and Technology Press, Wuhan, 2003.

[2] Gao Xueyao, Zhang Chunxiang. Explorations on Visual Teaching of Computer Graphics, J. Science and Technology, 22(2014)104.

[3] Wei Haitao, Zhao Zhizuo, Wu Caihua. Reform and Reflections on Computer Graphics, J. Industry and Information Technology Education, 12(2014) 49-54.

[4] Li Honglin. On Experimental Course Reform of Computer Graphics, J. Science \& Technology Information, 12(2014) 155-156.

[5] Yan Shenghua. Teaching Research and Practices on Computer Graphics, J. Computer Education. 13(2010)114-116.

[6] Zhao Qinping. A Summary of Virtual Reality, J. Science in China (Series F, Information Sciences). 39(2009) 2-4. 